\title{
THE REPAIR OF INJURIES TO THE PERINEUM AND VAGINAL WALLS.
}

\author{
BY FREDERICK J. McCANN, M.D., F.R.C.S., \\ Consulting Surgeon, Samaritan Free Hospital for Women.
}

The frequency with which injuries to the perineum and vaginal walls are discovered, not only after what is regarded as a normal labour, but still more after instrumental delivery, should compel all medical practitioners who are called upon to attend the parturient woman to master the principles that govern successful repair. The ease with which further advice may be obtained is no justification for the inability to deal with an urgent complication, and through lack of immediate treatment many women are condemned to months or even years of unnecessary suffering, suffering that could have been avoided by timely intervention. Still it is remarkable how Nature strives to remedy, with a varying amount of success, injuries that are not too severe, and for this reason many disabilities continue undiscovered until a local examination is made. It was the persistence of these disabilities that gave rise to the gibe that the obstetrician provides work for the gynæcologist. Even a perineal tear extending into the rectum may be so compensated that the woman is unaware that the bowel is injured, while examples of imperfect repair are the commonplace of medical practice. Indeed parturitional injuries untreated, or inefficiently treated, are the chief factors in the production of the morbidity of child-bearing, and the victims are numbered not by thousands but by tens of thousands, while their ailments seem to be forgotten in the hue and cry raised over maternal mortality of which the rate, when contrasted with that of maternal morbidity, is insignificant. This morbidity can be reduced by the successful treatment of parturitional wounds.

\section{Injuries to the Perineum.}

When the perineum is torn the adjacent vaginal wall is almost invariably included in the tear, but the lowest segment of the posterior vaginal wall may be torn without extension of the tear to the cutaneous surface of the perineum, and for this reason tears in this locality often escape recognition. The skin of the perineum having been found intact on inspection, it is assumed that no tear exists.

As it is imperative that all perineal tears should be repaired, it is unnecessary to perpetuate the distinction into three degrees depending upon the extent of the tear; first degree, superficial tear; second degree, reaching to the anus; and third degree, when the rectum is torn. This teaching stresses the importance of the rectal tear, while forgetting the serious disability which follows failure to repair injuries that do not extend into the bowel. To boast that in a large obstetric practice perineal stitches have rarely or never been required is not only an admission of negligence, but still more indicates a lack of appreciation of the morbidity that follows when perineal wounds are either neglected or are incompletely repaired. It is the duty of the doctor to explain to his patient the importance of repairing perineal wounds in order not merely to prevent future discomfort, but to obtain a restoration of normal structure. The practitioner, however skilled, cannot always prevent perineal tears, nor even their occasional extension into the rectum, but the repeated recurrence of extensive tears in the practice of the same doctor is a sure indication of the need for post-graduate instruction! 


\section{Cause of Perineal Tears.}

The cause of perineal tears is to be sought in the disproportion between the passages and the passenger, the narrow passages and the large child, in malpositions chiefly when the occiput is posterior, in malpresentations, in the aftercoming head, in the unskilful delivery of the child (manual and most often instrumental), and in roughness in the extraction of the placenta. Again a prolonged second stage or a rapid delivery are productive of a special type of injury to which further reference will be made, while the nature of the perineal tissues is of signal importance. Softness and friability, thinness and tenseness, toughness and inelasticity, these variations in the character of the perineal tissues render them prone to tear and should put the practitioner on the alert to prevent tearing.

\section{The Nature of the Injury.}

A study of the anatomy of the perineum will explain why injuries in this locality are followed by displacements and disorders of various kinds, for in the majority of these injuries (if at all extensive) damage is done to the musculoaponeurotic structures that join in the formation of the pelvic floor. This damage is repeatedly done at a vulnerable point, for the centre of the perineum is the meeting place of a series of muscles and their covering fascia, upon whose integrity the sphincteric control of the vagina and rectum chiefly depends. The constrictor vaginæ, transversus perinei, and sphincter ani muscles are merged in the centre of the perineum, while at a deeper level the levatores ani are found clothing the vaginal and rectal walls. Superficial tears involve vaginal mucous membrane, perineal skin, and some fibres of the constrictor vaginæ. Deeper tears involve more fibres of the constrictor vaginæ, fibres of the transversus perinei, and even superficial fibres of the sphincter ani. Still deeper tears involve the sphincter ani and open the rectum (anal canal), and, exceptionally, extend further upwards in the rectal wall, involving and displacing the levatores ani muscles. The levatores ani muscles are, however, more frequently displaced and over-stretched even where there is little or no perineal injury, thus leading to the production of the well-known gaping vagina whose walls ultimately descend or protrude. This over-stretching is often a sequel of a precipitate labour or of a rapid delivery, and in the majority of instances it is assumed by the patient that she has been torn, and for this the doctor is, not infrequently, unjustly blamed. A prolonged second stage may be associated with injuries of another character where the presenting part burrows into the vaginal wall behind the intact perineum and, in addition to overstretching and displacement of the levatores ani muscles, a cul-de-sac is formed through tearing of the lower end of the posterior vaginal wall, and this cul-de-sac unrecognised and untreated may, in the presence of infection, become a "pus pocket." An excessive amount of burrowing is met with in the rare examples of delivery through the centre of the perineum. Occasionally too, the rectum is opened into behind the perineum, and a fotal foot or arm has appeared through the anus.

\section{The Effect of Perineal Injuries.}

The effect of perineal injuries depends upon their extent and upon the manner in which they are treated. It is no exaggeration to state that the large majority of tears are left untreated because they are not recognised, but when the bowel is torn the effect becomes obvious, even to the most superficial observer, through incontinence of fæces. And in the absence of bleeding from the torn surface, 
which occasionally may be what is called "free" bleeding, little notice may be taken of other signs. Unfortunately the dire results of submucosal tearing, crushing, or separating muscles do not become obvious until the patient attempts to resume her ordinary duties, although a general sense of local weakness or loss of muscular power may have existed since the birth of the child. When the sphincter ani is torn there is, as already mentioned, incontinence of fæces. Yet even in the presence of a torn anal sphincter a certain measure of retentive power may in time be re-established, but the rectum is neither air-tight nor water-tight, in other words there is involuntary escape of flatus and of a watery stool. The healing of the torn bowel results in the formation of a transverse scar between the rectum and the vagina, with the remains of the torn sphincter on either side. It is because of semi-circular compression using the transverse scar as a point d'appui, aided in addition by pressing the gluteal muscles together in the middle line, that the existence of the defect may not be realised by the patient nor discovered by the doctor. In these circumstances the control may yet be both air-tight and water-tight, which is in marked contrast to the effect of a tear extending further upwards in the rectal wall where the loss of control is complete.

Lack of control may follow incomplete healing where an attempt to repair the bowel has merely resulted in the formation of a "bridge of skin" in the position of the anterior wall of the anal canal; the severed portions of the sphincter ani having either failed to unite or where an endeavour to unite them has not been made. Again even the "bridge of skin" may not be formed, and the loss of control may be increased still further through the unsatisfactory union of the severed tissues when the operation has failed in its purpose.

\section{Injuries to the Vulva and the Vagina.}

Injuries to the vulva usually result from the unskilled use of instruments, chiefly forceps, and occasionally from rough manipulation. The gradual disappearance of craniotomy, and other mutilating operations, from obstetric practice, has diminished the frequency with which injuries to the vulva occur. The labia minora may be abraded, compressed, and even torn, and tears may extend deeply on either side of the urethra dividing the blood vessels supplying the clitoris and be followed by considerable loss of blood. One or more of these accidents may result from the unskilled use of forceps, while more extensive injuries are discovered after pubiotomy in the hands of the inexperienced operator.

Stretching, crushing, bruising, tearing are seen both in the vulva and in the vagina and hæmatomata and sloughing are additional complications.

Although injuries to the lower end of the anterior vaginal wall apart from compression are relatively rare, yet " sagging" of the urethra and more especially of the external urinary meatus are common sequelæ of compression together with slight cystocele sometimes inducing incontinence of urine or more often frequent micturition and incomplete control of the sphincter. Tears of the vagina at a higher level are, as a rule, caused by forceps, and the subsequent hæmorrhage may give rise to the impression that the blood is coming from the uterus. But when the uterus is well contracted the source of the bleeding should be looked for in the perineum, in the vaginal walls, and in the cervix. The vagina may be torn in conjunction with the cervix or with rupture of the uterine wall as well as independently. A slight amount of tearing of the cervix is a normal happening, but when extensive the tears should be sutured and thereby prevent 
many subsequent ills. The cervix may, occasionally, be deeply torn most frequently on the left side, or the tear may be bilateral. A portion of the cervix may be torn loose or removed in the grasp of the forceps, or in exceptional cases the entire circumference is torn. I have seen a uretero-vaginal fistula complicate a "forceps injury" where not only a portion of the cervix had been torn but the right ureter had been nipped at the same time, leading to the formation of a fistula which had existed for twenty-five years when this woman was examined by me. Various unsuccessful attempts had been made to close the fistula but the closure was ultimately accomplished by a method originally described by me in the British Medical Journal (May I7th, I902).

In the absence of hæmorrhage a routine search for tears in the upper half of the vagina and in the cervix is to be deprecated for the added risk of infection must ever be present in the mind of the attendant practitioner, but it is well to look within the perineum, for the bruised and torn pocket so frequently discovered there is a potential danger spot, and is one of the chief sources of infection in puerperal sepsis, yet it is capable of being rendered innocuous by timely repair.

Unfortunately the most common injury is hidden, and on that account overlooked, for it consists in a submucous separation of muscular fibres with consequent loss of power and functional impairment, while the over-stretched elastic tissue of the vagina suffers in like manner.

In many instances there is a divarication of the levatores ani muscles that is to say they have been made to diverge, thus permitting the rectum to bulge into the separation so produced and form what is termed a rectocele. A somewhat similar effect is produced in the anterior vaginal wall when through severe compression and stretching the musculo-aponeurotic supports yield and permit the bladder (and with it the anterior vaginal wall) to descend and eventually to protrude forming a cystocele. Prolonged pressure on the anterior vaginal wall by the presenting part may be followed by bruising, tearing and subsequent sloughing, and I have seen a slough in the neighbourhood of the external urinary meatus, which on separation left a wide meatal orifice with subsequent incontinence of urine. Even from less severe compression the function of the bladder is affected so that the power of retention and expulsion are alike impaired.

Let me repeat that it is those injuries giving little or no superficial evidence of their existence yet causing submucous or subcutaneous bruising, stretching, tearing and dislocation of muscle and fascia that are productive of so many of the disabilities following childbirth. These disabilities continue unheeded until prolonged discomfort, pain or prolapse compel the sufferers to seek relief. The recovery from a "squeezed bladder" may occasionally be very slow due in all probability to the persistence of pressure paresis and this without any displacement of the bladder.

\section{Fistulæ.}

Fistulæ may be the result of pressure necrosis or of direct injury. Rectovaginal fistulæ and vesico-vaginal fistulæ, especially the former, are most common, while vesico-cervical and uretero-vaginal fistulæ are relatively rare. Fistulæ are not now so frequently met with as in former days, and despite the continued wail regarding the teaching and practice of obstetrics there has been a steady diminution in the number of women suffering from fistulæ or pressure necrosis with its attendant sloughing. I have seen some terrible effects of sloughing 
following the improper use of forceps; in one instance the whole of the vagina had sloughed, in another the anterior vaginal wall and the base of the bladder, and in yet another the greater part of the recto-vaginal septum as well as the base of the bladder and subjacent portions of the anterior vaginal wall, so that rectum, vagina, and bladder formed a common cloaca; whilst the subsequent cicatrisation that I have seen in those who have escaped death may be so extensive that even a vaginal examination is no longer possible. The marked decline in the number of cases of this character is an achievement of which little is heard, but it is convincing evidence that the art of obstetrics has undergone considerable improvement in recent years. The truth is that severe injuries are as a rule prevented but if perchance they do occur they are treated successfully. But damage less serious albeit crippling in its results is still far too common and it demands and should receive the attention of all those who are concerned with the management of childbirth.

\section{Treatment.}

(a).-Preventive.

(b).-Curative.

(a) Preventive. Much can be done to avoid injuries by the careful conduct of labour as well as by pre-natal supervision, still it is not my present purpose to dwell upon the general management of pregnancy and parturition, it is rather to stress the importance of certain details whose neglect may lead to accidents which are avoidable. To prevent a precipitate labour wherever possible and in any case to prevent the rapid escape of the head of the child and especially of the shoulders and to avoid injury to the soft tissues during any manipulations are obvious precautions. Furthermore, to prevent undue prolongation of the seconds stage of labour, yet at the same time to bear in mind the importance of allowing time for the head to mould before attempting to apply forceps are essentials. To acquire the art of using forceps without injury to mother or child is an absolute necessity remembering too that "delivery by brute force" is never justifiable. The need of strong traction efforts is a sure indication either that the forceps have been applied too soon (generally on an imperfectly moulded head or on an unrecognised malposition) or that the disproportion between the passages and the passenger is such that an alternative method of delivery is required. To remove the forceps after the head has been brought down to the perineum, or in other words to refrain from delivering the head by forceps traction is another advantageous precaution, yet with care and without haste injuries can be avoided even when the forceps are not removed. And above all to aid the slow stretching of the perineum is the best means of avoiding sudden rupture. The dangers of rough manipulation in the third stage of labour have already been mentioned, still the facility with which the stretched oedematous tissues are bruised or lacerated seems to be forgotten by those who are not endowed with a gentle touch, and at the present time when women are so frequently drugged during the fulfilment of what is after all their primal function, it is all the more necessary to guard against the possibility of injury to the unconscious or semi-conscious mother whose dulled sensations prevent her natural resentment.

(b) Curative. Although the same surgical principles are applicable in the treatment of perineal and vaginal wounds as in wounds in other localities in the body yet if special precautions are not taken to secure accurate healing and functional restoration the results are disappointing and for this reason reparative operations were for a long time neither held in high esteem by the rank and file of the 
profession nor sought by the public. It was not uncommon to be told by a doctor that an operation would be a failure, or by the laity that no operation could be done or if it were done that it would be a failure. Here again improvement in technique has restored the confidence both of the profession and of the public in these operations. And it is true to state that there is no operation in surgery which when successful brings more comfort and satisfaction to the patient than the cure of a recto-vaginal or a vesico-vaginal fistula while the treatment of the sequelæ of perineal and vaginal injuries when founded on sound surgical principles is a triumph of modern gynæcology. Nevertheless many attempts do not succeed or only meet with partial success because the chief aim of good surgery - the preservation of function-is disregarded.

\section{Perineal Injuries.}

The number of perineal injuries neglected or overlooked and left to chance is still too large, and greater attention should be paid to their recognition and prompt treatment. Treatment may then be applied either at the time of injury or at a later date.

\section{Immediate Treatment.}

There are few women in whom the birth of a child does not inflict a perineal wound, but this wound may vary in depth from a crack or fissure of the skin or mucous membrane to a tear extending into the bowel. The majority of tears however do not extend deeply and the bowel escapes injury. The common type involves the perineal body and extends into the vaginal wall usually on the left side. To repair this injury it is necessary to stitch the torn vaginal wall, to reconstruct the torn perineal body and to unite the torn skin. Two kinds of sutures are necessary, absorbable and non-absorbable, and it is a good rule never to trust absorbable sutures alone, for although a pious hope may be expressed on the glass tube in which they are contained that they will last ten, twenty, thirty, forty days this is not always a statement of fact, and sooner or later it will be realised by their premature absorption. Heat, moisture, and liability to infection militate against the continued success of catgut used alone in the perineum, and reinforcing sutures of a non-absorbable material are a necessity, and the transformation which the genital organs undergo during the puerperium may be an added factor in promoting rapid disappearance of catgut. Failure of union so produced is far from uncommon and may become a cause of action in the Law Courts. And in a case in which I was called as an expert witness a complete perineal tear had been stitched with catgut and failing to unite incontinence of fæces persisted and an action for negligence was brought against the doctor.

An immediate operation is recommended to be performed during the third stage of labour before the placenta is expelled, or stitches are inserted and left untied until the expulsion of the placenta. But this leads to slipshod work, and it is worth while to aim at obtaining accurate apposition in the beginning undisturbed by manipulations in the third stage of labour or even by the expulsion of the placenta and membranes. There is no advantage to be obtained by leaving sutures untied, for it is advisable in any case to administer an anæsthetic and to do the work thoroughly. The woman should be placed on her back and in a good light in order to see the extent of the injury and for this purpose the wound should be opened out by a pair of Kocher forceps applied on each side to the skin edge. If the rectum be uninjured the vaginal mucosa should be stitched with interrupted deep and superficial catgut sutures, the deep sutures to co-apt the torn muscles. The torn perineum should be stitched with interrupted silkworm gut sutures inserted a 
quarter of an inch from the skin margin by a medium-sized curved needle on a needle holder, (or pair of artery forceps if no needle holder be available), and a good grip of the tissues having been taken the needle is brought out at the deepest part of the wound; it is then pulled out by forceps or needle holder and introduced on the other side (again deeply) from below outwards and upwards, taking a good grip of the tissues to emerge through the skin a quarter of an inch from the margin at a corresponding point to that of entry on the opposite side. It is important to appreciate the manner in which these sutures are passed, for hitherto the almost universal practice has been to use a large curved needle on a handle, or grasped by a needle holder and passing the needle deeply (still buried) it is brought out through the skin on the opposite side. When a stitch so inserted is tightened it is merely a purse-string suture and results in bringing the deep portion of the wound in closer contact with the surface which is what should be avoided. A perineal wound should be treated in the manner of a divided anterior abdominal wall and here a suture so inserted as to bring the peritoneum closer to the skin of the abdominal wall would not commend itself to anyone possessing surgical nous. It is then easy to understand how a faulty method of suturing the perineum favours the subsequent formation of a rectocele, a complication which is (or should be) the aim of the surgeon to avoid.

A special effort is also made to unite torn muscles and fascia for although the silkworm gut sutures suitably inserted may do this, still, it makes for greater accuracy to stitch the muscle. And it must not be forgotten that sutures do not unite wounds; they merely act as splints until Nature glues the edges together. For this reason sutures must not be pulled too tight (especially non-absorbable) and thus it is necessary to acquire the art of apposition without constriction, for ? subsequent œedema may readily transform a suture inserted and tied carefully into one that causes pain and discomfort. Two or three sutures should be passed to unite the torn muscular fibres in the perineal body and thereafter the perineal skin is united by interrupted catgut sutures before the silkworm gut is tied. Continuous sutures should be avoided because they are less secure. If the torn edges are ragged they should be trimmed with a sharp knife while all the blood and blood clots should be removed before the wound is sutured. And a plug of gauze wrung out of $I$ in 2000 biniodide of mercury should be placed in the vagina to prevent blood from the uterus flowing over the wound during the operation. This plug is removed after the suturing is complete. When the lower end of the posterior vaginal wall is torn and the perineum split leaving the skin surface intact the best method of repair is to divide the perineal skin in the middle line thereby opening up the wound and then to suture in the manner already described. This method is both easier and more efficient than that of attempting to complete the operation within the perineal skin while it has the further advantage that you can see the depth and character of the tear and for this reason the insertion of the sutures can be more accurate. The importance of the repair of this particular type of injury cannot be overestimated for to the risks of infection there is superadded the subsequent disabilities due to the musculo-fascial tears and yet this is, as previously stated, the injury that is most frequently overlooked because the perineal skin having been found intact it is assumed that no tear exists. An infected perineal wound is the source of inany local and general infections during the puerperum and it is this kind of wound which is the most dangerous, for hidden behind the perineal skin a "pus pocket"may form and in any case infection is readily carried to a higher level in the genital canal through manipulation or instrumentation. 
In the ætiology of puerperal infection the significance of the wounded perineum as the original site has been under estimated. It is well-known that in the skin a scratch or a pinprick may be followed by the most virulent septicæmia yet the scratches and tears in the perineum and vagina are often ignored while a mass attack is made on the uterus which in all probability is infected by this alone, more commonly than is supposed.

\section{Rectal Tears.}

The immediate suture of a rectal tear fails in a large proportion of cases and as many as two-thirds are reported as failures by some writers. Minute attention to technical details is the only means of improving the results and of restoring function. The chief causes of non-union or imperfect union, apart from sepsis, are inaccurate suturing with unreliable material, which either fails to hold by cutting out or becomes absorbed too quickly or the neglect to arrest all bleeding and to trim ragged edges with a sharp knife, but of this anon.*

\section{Repair of Perineal Injuries at a later date.}

If a rectal tear has been overlooked in the beginning or has been incompletely repaired or has "broken down" the question that leaps to the mind is at what date should a further attempt be made to repair the torn bowel? There seems to be such a lack of agreement on this question that some obstetricians would even permit a woman to suffer the disagreeable consequences of partial or complete loss of the control of her rectal sphincter for several months on the assumption that an earlier attempt would certainly fail. Lactation is not a hindrance but what really matters is the condition of the wound. If the condition of the wound be favourable the sooner the injury is repaired the better, and if it be unfavourable then remedial efforts should be made without delay in order that the wound may be rendered suitable for a trial to be made to re-establish sphincteric control. I have successfully repaired perineal injuries at all dates in total disregard of the injunction that weeks or months should elapse after childbirth before a further attempt is made. The art of modern surgery would be poor indeed if a woman were compelled to continue to endure this distressing physical damage because of some imaginary hindrance to the success of early surgical treatment, which is in reality an unfounded belief perpetuated from earlier days. The discrepancy in the statements of the medical witnesses in the action for negligence to which $I$ have referred, as well as in the statements appearing in modern medical books concerning the date when an operation should be attempted, has prompted me to lay stress on the significance of early operation (whenever possible) in the interests of the patient, for even a granulating wound may be re-sutured with success.

The presence of scar tissue in the vaginal wall and between the torn bowel and vagina, a sequel either of the original injury or of attempts to repair it, is a complication which serves to explain in many instances the subsequent failure of reparative operations. Scar tissue must be excised, for union will not be obtained where the remnants of a scar still adhere to the margin of the sutured rectal wall. The failure of union at the upper extremity of a rectal tear with the subsequent formation of a recto-vaginal fistula is in most cases due to the incomplete removal of scar tissue, as well as to inaccurate suturing in particular under tension. For this reason it should also be recognised as one of the most important principles to ensure by free separation of the tissues implicated that there is no tension when the stitches in the rectal wall are inserted and tied. With this aim

*The practitioner must endeavour to obtain reliable catgut. No. 1 for the bowel, No. 2 for the skin and mucous membrane, No. 3 for muscles and fascia. 
in view the lower end of the rectum must be detached in front and at the sides. The lateral separation should further expose the anal sphincter so that complete sphincteric control may be re-established by suture of the torn muscle and the former anatomical position restored, namely, surrounding the anal orifice. The description in many books of how to unite this torn muscle would be quite amusing were the consequences of failure less disastrous. A single stitch is used to bring the torn circular muscle into the correct anatomical position and illustrations are published to show how this should be done and much ingenuity is wasted in fruitless efforts to devise a special type of suture. When stitching the rectum it is a good plan to begin by passing one or two stitches through the rectal wall (avoiding the mucosa) above the upper extremity of the tear, for when these are tied a ridge is formed which not only facilitates the introduction of the remaining stitches but also favours the union of the upper end of the tear, which is the point where a fistula is most likely to develop. The rectal sutures are inserted from above downwards and should take a grip of the wall of the bowel avoiding the mucosa, and be tied in the lumen of the bowel. The edges of the tear are thus inverted and if a sufficient grip has been taken by the needle the inverted edges are brought into contact and a ridge (which subsequently disappears) is formed along the anterior wall of the anal canal. The principle is that of the Lembert suturewell-known in the surgery of the intestine-and it is this kind of suture that gives the best results when stitching rectal tears. Failure of union may be, and often is, the direct outcome of the neglect to obtain inversion of the edges of the torn bowel, through the inadequate amount of tissue held in the grasp of the suture, for it does not suffice merely to bring the edges together; they should not only be inverted but enough of the rectal wall should be apposed on each side to ensure firm union. In short it is the "niggling operation" that is doomed to be a failure. The torn sphincter muscle is best repaired by the method of stitching that is used for the rectal wall, but here also a good grip must be taken of the sphincter muscle (which can be seen where the lower end of the rectum is separated) while a sufficient number of stitches is required to make certain that the sphincter muscle has been replaced and that it completely surrounds the lower end of the anal canal. There is no fear of constricting the anal orifice if you operate anatomically, for it is the formation of scar tissue following ineffectual and haphazard suturing that causes stricture. And it is, I repeat, essential to avoid tension on the sutures by free lateral separation of the rectum in the first instance and further snipping where there is still evidence of tension on the sutures when they are tied. The secret of success is to free the lower end of the rectum in front and at the sides for even if the sutures prove unreliable there is yet a good prospect of subsequent union. The sphincter muscle must be thoroughly exposed on each side of the anal orifice, for when the muscular fibres are rendered visible they can be sutured without difficulty in the middle line and a strong sphincter constructed, which is in marked contra-distinction to haphazard stitching when the muscular fibres remain buried. It is through the neglect of these technical details that the vast majority of these operations fall short of their purpose, leaving the patient little better or often worse than before. There is a tendency, at least in extensive rectal tears, for the reconstructed anus to fall backwards towards the sacrum. In order to prevent this-the "sacral anus"-it is desirable to restore the forward as well as the downward curve of the anal canal. This can be accomplished by a pursestring suture which is passed through the skin close to the anus on one side, then under the sphincter ani and through a portion of the levator ani and in a reverse direction on the other side through the levator ani and under the sphincter ani to pierce the skin close to the anus on the same side. When the stitch is tied it will 
be found that the normal curve of the anal canal has been reproduced. When the anus is reconstructed there should be evidence of a return of the normal contour for, if not, it is safe to assume there is some technical fault. Let me reiterate that the failure to restore the power of the anal sphincter is due to the non-union or partial union of the sphincter ani, for functional restoration depends upon the thoroughness with which the repair of the sphincter ani has been effected. It is not possible in the vast majority of cases to obtain restoration of function by one stitch alone and the promulgation of the "one stitch idea" may possibly account for a large proportion of the failures. The sphincter muscle should be exposed thoroughly and co-apted by a sufficient number of stitches to fix it in the normal position, and it is only by a thorough exposure of the anal sphincter that the full extent of the injury can be determined.

These then are the principles which underlie successful repair, and yet I have not even mentioned the kind of incision to be used because in this department of surgery too much stress is laid on patterns and patchwork and too little on principles. The incisions to be made should be those best suited to attain the objects in view. In the majority of cases a short transverse incision splitting the rectum from the vagina; at each extremity prolonged downwards and backwards to expose the sphincter ani and also upwards and forwards for a varying distance into each labium, meets all requirements. The anterior prolongation enables a suitable vaginal flap to be raised and further enables the rectum to be detached freely from the vaginal wall. The vaginal flap should as a rule be allowed to remain intact unless there is a cicatrix on it, and if there is, the cicatrix should be excised and the wound repaired. The restoration of the perineal body which is the keystone of the arch is of prime importance. Here it is essential that a solid mass of tissue should be built up in the interval between the lower end of the vagina and the lower end of the rectum.

To do this it is further essential that the musculo-aponeurotic structures entering into the formation of the perineal body should as far as possible be restored to their normal position. This is best accomplished by sutures of apposition and non-absorbable sutures of support. The non-absorbable sutures of silkworm gut are passed, as already stated, on a curved needle through the skin of the perineum a quarter of an inch from the cut margin and taking a good grip of the underlying tissues are brought out at the deepest part of the wound to be reintroduced from below on the opposite side and again taking a good grip are made to emerge through the skin a quarter of an inch from the cut margin. Sutures thus introduced do not bring the bottom of the wound to the surface and in consequence they aid in the reconstruction of a solid mass of tissue in the position of the perineal body.

But there is still an important principle which should not be forgotten in the conduct of these operations, and that is to provide in all situations a second line of defence. This is the chief reason why a non-absorbable suture is advantageous in perineal surgery.

The levatores ani muscles should be brought together over the rectum and for this purpose catgut sutures may be employed three or four in number and beginning from below upwards they should take a good grip of the levator ani muscle which is readily identified on each side of the wound. These sutures when snugly tied assist in re-building the musculo-aponeurotic barrier which is the chief component of what is generally known as the perineal body. It now only remains to unite the skin of the perineum by interrupted sutures of catgut and after these have been tied the silkworm gut sutures are knotted with a surgeon's knot but are 
not pulled tight: in other words the sutures bring the edges of the skin into apposition but they do not constrict. The use of small pieces (half an inch) of thin rubber tubing through which the threads are passed is an advantage not merely in avoiding constriction but in preventing burying of the suture both of which are apt to lead to difficulty in the removal of the sutures and to cause unnecessary suffering. The silkworm gut sutures are tied together in order to facilitate their subsequent removal and at the same time to prevent pricking of the skin which is not infrequent when the ends are cut short. These sutures being indispensable as splints, should not be removed for ten, twelve, or fourteen days. Even if the wound be infected and "break down" it is wise to leave the sutures undisturbed while efforts are being directed towards disinfection. Attempts are made to force the hand of the doctor (and they should be resisted) by demanding the early removal of the sutures because; they are alleged to be causing "irritation" ; are no longer of any use; are preventing healing; or are "infected". All this should merely serve to impress upon the mind the great value of the silkworm gut suture for it continues to be a splint and it does not cause "irritation" when properly tied, while it helps to hold wound surfaces together even in the presence of infection; moreover it does not become infected and it can, without difficulty, be sterilised before use. It cannot be denied that silkworm gut sutures tied too tightly (and the tightness increased by subsequent œedema) do cause considerable pain and "irritation" but this important fact should serve to impress the necessity for greater technical skill in the use of this material. And although such faulty suturing may necessitate the premature removal of one or more of the sutures yet the fault lies not with the material used but with the manner of its use.

To remove sutures of this kind (introduced with the proper safeguards) on the assumption that they are causing delay in healing is not merely a technical error through removing the scaffolding before the reinforcing structure is complete, but it may lead to further disaster when the wound gapes for then the local state is worse than it was in the beginning, and the loss of muscular power and control is greater. But the healing may be partial, resulting in a weakened sphincter through inadequate or absent muscular union, or a recto-vaginal fistula may subsequently give evidence of its presence by the passage of flatus and fæces through the vagina.

In order to close a fistula in this situation the tissues intervening between the fistulous tract and the anus should be freely divided and an operation on the lines already indicated should then be performed. Paring the margin of the orifice of the fistula followed by suturing is generally a failure, and is, moreover, inadvisable on account of the frequency with which defective musculoaponeurotic union co-exists. When a "paring operation" succeeds it is good luck rather than good surgery. The same is true of vesico-vaginal fistulæ and here extensive flap splitting and freeing of the bladder wall in the locality of the fistula so that sutures may be inserted and tied without undue tension upon them gives the best results. Let me repeat in conclusion that it is essential to master the principles which should govern attempts to repair the damaged tissues, and when these principles are applied in the different localities a degree of success can be obtained that is unsurpassed in any other branch of surgery.

Note.-For the treatment of the gaping vagina, the result of sub-mucosal stretching and tearing, the reader is referred to my book on "The Treatment of Common Female Ailments," second edition, Arnold \& Co., Maddox Street, W., wherein the treatment of puerperal infection is also considered. 